\title{
Thermal conductivity of Xe clathrate hydrate at low temperatures
}

\author{
A. I. Krivchikov,* B. Ya. Gorodilov, O. A. Korolyuk, V. G. Manzhelii, and O. O. Romantsova \\ B. Verkin Institute for Low Temperature Physics and Engineering of NAN Ukraine, Kharkov, Ukraine \\ H. Conrad \\ Institut fur Festkorperforschung, Forschungszentrum Jülich GmbH, 52425 Jülich, Germany \\ W. Press \\ Institut Laue-Langevin, Grenoble, France \\ J. S. Tse \\ Department of Physics and Engineering Physics, University of Saskatchewan, Saskatoon, Canada S7N 5E2 \\ D. D. Klug \\ Steacie Institute for Molecular Sciences, National Research Council of Canada, Ottawa, Canada K1A OR6
}

(Received 14 June 2005; revised manuscript received 5 January 2006; published 7 February 2006)

\begin{abstract}
The effective thermal conductivity of the powder samples of xenon hydrate was measured in the interval $2-170 \mathrm{~K}$ using the steady-state method. The thermal conductivity of the homogeneous Xe clathrate hydrate was estimated from the effective thermal conductivity using an empirical expression. The applicability of the formula was checked by comparing two powder samples with different grain size and porosity. The temperature dependence of the thermal conductivity $\kappa(T) \sim T^{n}$ of Xe clathrate hydrate is divided into four distinct temperature regimes (I-IV) with different $n$. In the interval 55-97 K (III) the behavior of $\kappa(T)$ shows an anomaly, where the thermal conductivity decreases by almost $50 \%$ as the temperature increases. This observation is attributed to the resonant scattering where the coupling of the lattice with "rattling" motions of Xe atom dominates the thermal resistivity at high temperature. Since the observed vibrational energy of Xe in the small cages is $\sim 4 \mathrm{meV}$ ( or $\approx 46 \mathrm{~K}$ ) the resonant scattering contribution to the thermal resistivity is expected to decrease in an interval of comparable temperature. The thermal conductivity in the low temperature regime (regimes I and II) is found to follow the prediction of the soft-potential model. The data on thermal conductivities of several gas clathrate hydrates are compared.
\end{abstract}

DOI: 10.1103/PhysRevB.73.064203

PACS number(s): 66.70. $+\mathrm{f}, 63.20 .-\mathrm{e}, 63.20 . \mathrm{Pw}, 63.50 .+\mathrm{x}$

\section{INTRODUCTION}

Clathrate hydrates are open polymorphous crystal structures related to ordinary ice. Most of the thermodynamic and dynamic properties of gas clathrate hydrates are similar to the properties of crystal ice $\mathrm{I} h$. However, in 1981 Ross et al. ${ }^{1}$ found that the thermal conductivity of clathrate hydrates differed drastically in value and temperature dependence $\kappa(T)$ from that of ice. The very low thermal conductivity of clathrate hydrate decreases with lowering temperature and is only weakly dependent on the pressure. Later, the thermal conductivity of the gas clathrate hydrates was investigated by several research groups. ${ }^{2-5}$

The measurement of the thermal conductivity of 1,3dioxolane hydrate in an interval of $2-200 \mathrm{~K}$ has shown that such a thermal conductivity is typically observed for amorphous substances. ${ }^{3}$ After decreasing with temperature the curve $\kappa(T)$ has a plateau in an interval of temperatures comparable to that of glasses. Similar behavior of $\kappa(T)$ was also found in THF hydrate. ${ }^{4}$ The effect of proton ordering on the thermal conductivity of THF clathrate hydrate doped with $\mathrm{KOH}$ impurity was reported. ${ }^{5}$ Recently reliable data on thermal conductivity have been measured on gas hydrates of methane $^{6}$ and THF (Ref. 7) ( $2 \mathrm{~K}$ to $\sim 200 \mathrm{~K}$ ), which have different types (I and II, respectively) of clathrate structure.
In a wide range of temperatures the curve $\kappa(T)$ of the gas hydrates is only weakly dependent on the type of guest particle or clathrate structure and exhibits a behavior typical of amorphous or disordered solids. This implies that although clathrate hydrates are crystalline, their thermal conductivity follows a glasslike pattern. While on average with a welldefined crystal structure the hydrate displays considerable disorder. There are two types of disorder: (1) a proton disorder in the host lattice and (2) an orientational disorder of the guest molecules that occupy the cavities of the clathrate. Clathrate hydrate of inert gas can be used as a simple model for investigating the mechanisms of phonon scattering in other clathrate systems. In these substances the guest molecules have no rotational degrees of freedom and thus experience only translational oscillations (rattling motions) in the cages of this clathrate. The translational vibrations of the guest molecules are anharmonic ${ }^{8}$ and are coupled directly to the phonons of the clathrate framework. ${ }^{9}$ The degree of anharmonicity and coupling depends strongly on the type of guest molecule. ${ }^{8}$ The glasslike behavior of the thermal conductivity also was detected in some doped semiconductors $^{10-12}$ and organic ${ }^{13,14}$ clathrates.

In this study the thermal conductivity of Xe clathrate hydrate has been investigated and the origin of the noncrystal- 
line behavior of the thermal conductivity of crystal hydrates discussed.

The thermal conductivity of two clathrate hydrate samples $\mathrm{Xe} \cdot z \mathrm{H}_{2} \mathrm{O} \quad(z=5.9$ and 6.2 $)$ was measured in the range $2-170 \mathrm{~K}$ by the steady-state method. $\mathrm{Xe} \cdot z \mathrm{H}_{2} \mathrm{O}$ has a cubic $P m 3 n$ lattice (type I clathrate structure) with a lattice constant $a=12 \AA$. The stoichiometric clathrate structure of type I is usually denoted as $2 \mathrm{X} \cdot 6 \mathrm{Y} \cdot 46 \mathrm{H}_{2} \mathrm{O}$, which indicates that the elementary cell consists of 46 water molecules and contains 6 large tetrakaidecahedral and two smaller hexakaidecahedral cavities. Large and small cavities each can house one guest particle. In Xe clathrate hydrate the concentration of guests in the clathrate cavities can reach $98 \%$ under certain conditions of sample preparation. Hence the departure from stoichiometry may be small, as in the case of methane hydrate. The comparative analysis of the thermal conductivities of two hydrates with different guest molecules can provide additional information about the mechanisms of phonon scattering in crystal hydrates.

\section{EXPERIMENTAL TECHNIQUE AND SAMPLE PREPARATION}

Measurement of the thermal conductivity on gas clathrate hydrates poses a certain methodical challenge. These substances cannot exist under normal conditions (room temperature, atmospheric pressure). Above 150-250 K (depending on gas contents), gas clathrates decompose under equilibrium gas pressure, releasing gas and producing ice. In laboratories, gas hydrates are prepared by saturating fine disperse ice powder $(1-200 \mu \mathrm{m}$ grain size) with gas under excess pressure. The gas hydrates are kept and transported at liquid $\mathrm{N}_{2}$ temperatures.

Powder hydrates have fine-grained porous structures. The thermal conductivity of granular samples is dependent on the thermal conductivity of the material itself and on the thermal resistance between the grains. In turn, the thermal resistance has contributions from thermal contact resistance and from resistance of gas in intergranular spaces. The use of a heat exchange gas (helium) in the intergranular space allows to substantially reduce the relative contribution of the intergranular thermal contact resistance to the effective thermal resistance of the powdered sample over a wide range of temperatures. ${ }^{15}$

Since measurement of the true thermal conductivity $\kappa(T)$ of the material was problematic, we measured the effective thermal conductivity $\kappa_{\text {eff }}(T)$ of a powder sample with presence of ${ }^{4} \mathrm{He}$ gas and extracted the bulk thermal conductivity of the gas hydrate. A special setup ${ }^{16,17}$ was constructed for low temperature measurements of the thermal conductivity of clathrate hydrates by the thermal potentiometer method.

The sample container is a stainless steel tube $40 \mathrm{~mm}$ long and $22 \mathrm{~mm}$ in diameter with a the wall thickness of $0.3 \mathrm{~mm}$. The bottom of the container was fixed in the cooled zone of the cryostat connected to a helium bath. Two copper wires, $1 \mathrm{~mm}$ in diameter, pass through the container perpendicular to its axis, which permitted measurement of the average temperature along the isothermal plane running across the sample. The wires were $12.3 \mathrm{~mm}$ apart along the container
TABLE I. Sample parameters. $\eta$-density of the material package, PT-Preparation technology: 1- National Research Council of Canada; 2- Geozentrum Göttingen, University of Göttingen in Germany.

\begin{tabular}{cccc}
\hline \hline Sample & Grain size & $\eta$ & PT \\
\hline 1. Xe·5.9 $\mathrm{H}_{2} \mathrm{O}$ & $1-3 \mu \mathrm{m}$ & 0.18 & 1 \\
$2 . \mathrm{Xe} \cdot 6.2 \mathrm{H}_{2} \mathrm{O}$ & $0.1-1 \mathrm{~mm}$ & 0.56 & 2 \\
\hline \hline
\end{tabular}

axis. At the outer surface of the container, copper sockets were soldered to the wires to capsules of two temperature sensors. The upper sensor is a Cernox-SD resistance thermometer (Lake Shore Cryotronics, Inc.) measuring the temperature difference; the lower sensor is a TSU-2 resistance thermometer (VNIIFTRI) used to stabilize and control the temperature. The container with the sample was covered with an In-sealed vacuum-tight copper cap. A heater was mounted on the cap, allowing to generate a heat flow over the sample.

The statistical error in the effective thermal conductivity coefficient was no more than $3 \%$ in the whole range of measuring temperatures. The total measurement error $(\sim 10 \%)$ was mainly connected with the systematic error in measurement of geometrical parameters (inner container cross section, spacing between thermometers).

Two laboratory-made samples of clathrate hydrate were prepared using different technologies. Their main characteristics are given in Table I.

Sample 1 is a laboratory-made Xe clathrate hydrate (Xe.5.9 $\mathrm{H}_{2} \mathrm{O}$ ) prepared from the material supplied by the National Research Council of Canada. The material was a loose finely dispersed powder obtained by saturating ice $\mathrm{I} h$ with $\mathrm{Xe}$ gas under elevated $(\sim 10 \mathrm{bars})$ pressure at $T$ $=268$ to $283 \mathrm{~K}$ for 5 days. In the process of saturation, the ice was crushed continuously to particle dimensions of $1-3 \mu \mathrm{m}$. The density of $\mathrm{Xe}$ atoms in the cavities of the hydrate was $\sim 98 \%$. On completing the saturation, the material and remaining Xe gas were cooled down to liquid $\mathrm{N}_{2}$ temperatures and kept in this state until measurement was started. The pure Xe still available in the material was removed by heating the sample to $163 \mathrm{~K}$ (above the melting point of Xe).

Sample 2 was a laboratory-made Xe clathrate hydrate (Xe.6.2 $\mathrm{H}_{2} \mathrm{O}$ ) prepared from the material of GZG, University of Göttingen in Germany. This was a sintered disperse powder obtained from fine-grained $(10-100 \mu \mathrm{m})$ ice $\mathrm{I} h$ by the technique commonly used for gas clathrate hydrates. In the process of synthesizing Xe clathrate hydrate at $264 \mathrm{~K}$, the dispersed ice transformed in the Xe gas atmosphere (19 atm) into disperse crystal hydrate with crystallites of $20-30 \mu \mathrm{m}$. The initial fine disperse powder was sintered and a coarsegrained power was thus produced with the grain size not exceeding $1 \mathrm{~mm}$. The concentration of Xe atoms in the hydrate cavities was about $92 \%$. After removing the excess Xe gas from the space over the hydrate material, the sample was cooled to nitrogen temperature.

The gas hydrate sample was mounted in the cell for measuring the thermal conductivity. The clathrate hydrate pow- 


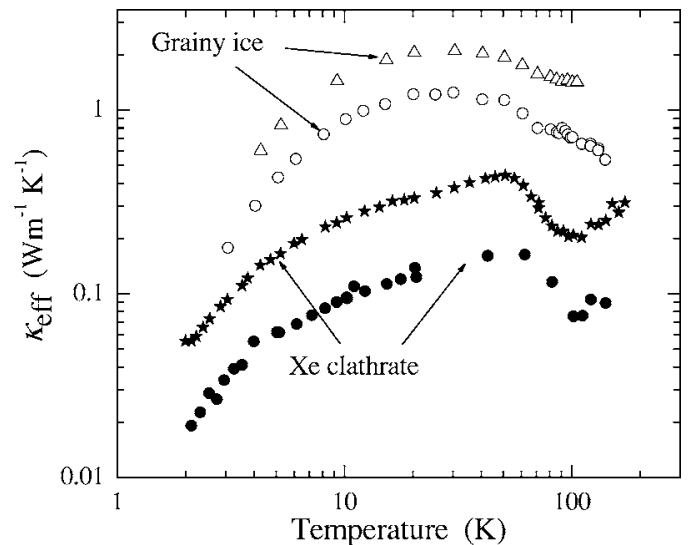

FIG. 1. The effective thermal conductivity of grainy Xe clathrate hydrate ( Sample 1, $\star$ Sample 2) and grainy ice after the separation of the clathrate hydrate sample $(\bigcirc, \triangle$ correspond to Samples 1 and 2 respectively).

der precooled with liquid nitrogen was fed into the container in small portions. In the process of filling the container, the sample was blown over with ${ }^{4} \mathrm{He}$ gas flowing from above. The temperature of the container was controlled continuously during the filling and subsequent manipulations. The temperature of the sample was not allowed to raise higher than $180 \mathrm{~K}$ to prevent decomposition of the gas hydrate.

\section{EXPERIMENTAL RESULTS}

The effective thermal conductivity of the two Xe clathrate hydrate samples was measured from $2-170 \mathrm{~K}$ (Fig. 1). After the measurement, each sample was allowed to decompose into disperse ice and $\mathrm{Xe}$ gas. The ice had a granular structure (grainy ice) similar to that of the initial material. The density of the material package $\eta$ (the ratio of the homogeneous sample volume $V_{s}$ and the empty-container volume $V_{c}$ ) was

$$
\eta=\frac{V_{s}}{V_{c}}=\frac{m_{H_{2} O}}{\rho V_{c}},
$$

where $m_{\mathrm{H}_{2} \mathrm{O}}$ is the mass of water and $\rho=0.912 \mathrm{~g} / \mathrm{cm}^{3}$ is the density of pure gas clathrate hydrate ${ }^{17}$ found from the weight of water present in the container after the measurement and heating to room temperature (see $\eta$ values in Table I).

The effective thermal conductivities $\kappa_{\text {eff }}(T)$ of two Xe clathrate hydrate samples and two grainy ice samples obtained after decomposition of Samples 1 and 2 were measured. The results of $\kappa_{e f f}(T)$ differ in value but have similar temperature dependences. The effective thermal conductivity of Sample 2 exceeds that of Sample 1 two times at the same temperature. The discrepancy obviously is due to different densities of package in the container and is independent of the preparation technique.

Below $40 \mathrm{~K}$ the temperature dependence of the effective thermal conductivity $\kappa_{\text {eff }}(T)$ of all samples is monotonous and does not have features suggesting either a significant adsorption of helium by the sample or formation of a superfluid He film on the grain surfaces. In one of the experiments we decided to measure the effective thermal conductivity of

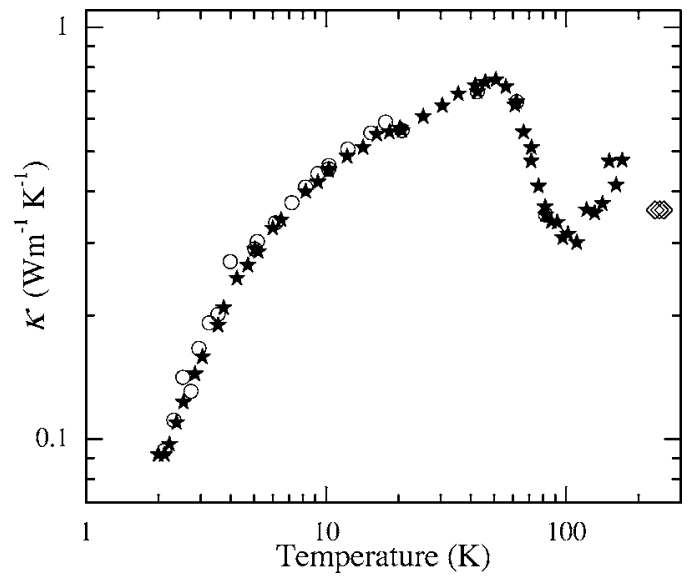

FIG. 2. Thermal conductivity of homogeneous Xe clathrate hydrate: $\bigcirc$ Sample 1; $\star$ Sample 2 [after correction with Eq. (2)]; and $\diamond$ Ref. 20.

Sample 2 in the absence of heat-exchanging He gas in the container. This value is an order of magnitude lower than $\kappa_{\text {eff }}$ of the same sample in the presence of the heat-exchanging gas. This difference existed over a wide range of temperatures.

The thermal conductivity coefficient $\kappa$ of solid homogeneous material was obtained from the effective thermal conductivity $\kappa_{\text {eff }}$ and the thermal conductivity coefficient of $\mathrm{He}$ gas $\kappa_{\mathrm{He}}$ (the grain-boundary thermal resistance is not included) using the following equation: ${ }^{15}$

$$
\kappa_{\text {eff }}(T)=\kappa(T) \cdot \eta+\kappa_{H e}(T)(1-\eta) .
$$

Equation (2) relating the effective $\left(\kappa_{\text {eff }}\right)$ and true $(\kappa)$ thermal conductivities was derived assuming that the heat flow was parallel to the layers of two substances - the material and the heat exchanging gas. Simple Eq. (2) is widely used to describe the thermal conductivity of powder materials. ${ }^{15,18,19}$ At the same time Eq. (2) is empirical and does not account explicitly for the grain structure of the substance and thermal contact resistance. These facts call for a check of applicability of Eq. (2) in our case. For this purpose we compared the thermal conductivities of homogeneous materials $\kappa$ estimated from the experimental data on the effective thermal conductivity $\kappa_{e f f}$ of Samples 1 and 2 using Eq. (2) and the $\eta$ values from Table I. Recall that Samples 1 and 2 are significantly different in preparation technique, grain size, and density of the material package $\eta$ (Table I). It is natural that the thermal contact resistances and the contributions of the heatexchange gas to the heat transfer are appreciably different in Samples 1 and 2, too. However, as follows from Fig. 2, the extracted thermal conductivities of homogeneous materials coincide for Samples 1 and 2 within the experimental error.

The coincidence of the true thermal conductivities of two gas clathrate hydrate samples (Fig. 2) suggests convincingly that the results for the thermal conductivity of homogeneous Xe clathrate hydrate are reliable. Our results extrapolated to the temperature interval above $200 \mathrm{~K}$ are a little higher than those obtained for the Xe clathrate hydrate prepared from compressed fine disperse granular material. ${ }^{20}$ 

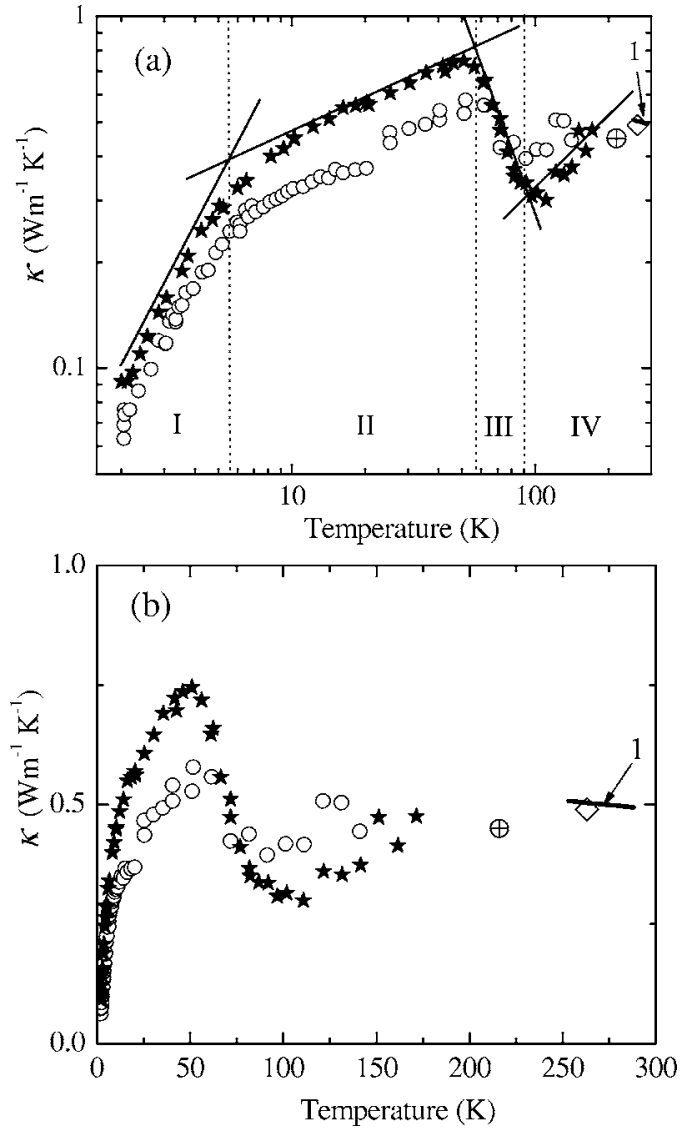

FIG. 3. Thermal conductivity of Xe clathrate hydrate ( $\star$ Sample 2) and $\mathrm{CH}_{4}$ hydrate: $\bigcirc$ Ref. 6; $\oplus$ Ref. 21; $\diamond$ Ref. 17; solid line 1, extracted data from $\kappa_{\text {eff }}$ (Ref. 22) of methane hydrate with $\mathrm{CH}_{4}$ gas using Eq. (2) in logarithmic (a) and linear (b) scales. Solid line on (a) is $\kappa(T) \propto T^{n}$ in the temperature range marked off by dotted lines. $n$ is given in Table II.

It should be noted that the curve $\kappa(T)$ of Xe hydrate has a feature which was not observed in other clathrate hydrates: the thermal conductivity increases as the temperature is lowered. Figure 3 illustrates the temperature dependences of the thermal conductivity of $\mathrm{Xe}$ and $\mathrm{CH}_{4}$ hydrates obtained by different techniques on porous samples of different finegrained porous structures. The porosity of $\mathrm{CH}_{4}$ hydrate samples prepared by compressing the initial material under external pressure up to 1 kbar was about $7 \% .{ }^{21}$ The data in Ref. 22 were derived from the effective thermal conductivity of the $\mathrm{CH}_{4}$ hydrate sample with $33.8 \%$ porosity (the samples was in a $\mathrm{CH}_{4}$ atmosphere) by Eq. (2).

In general, the temperature dependence of the thermal conductivity of homogeneous $\mathrm{Xe}$ and $\mathrm{CH}_{4}$ clathrate hydrates is described by the intervals with a similar power exponential relations $\kappa(T) \propto T^{n}$ (see Fig. 3).

Four intervals can be separated, each having constant $n$. The boundary temperatures of the regimes and exponents $n$ are shown in Table II and Fig. 3.

The temperature dependences $\kappa(T)$ in the intervals I, II, and IV are similar to what is observed in THF (Ref. 7) and dioxolane clathrate hydrates. ${ }^{3}$ The thermal conductivity grows with temperature and the curve $\kappa(T)$ has a shape typical for amorphous substances.
TABLE II. Powers $n$ in the power law behavior $\kappa(T) \propto T^{n}$ of the thermal conductivities of homogeneous $\mathrm{Xe}$ and $\mathrm{CH}_{4}$ hydrates for different temperature intervals.

\begin{tabular}{cccc}
\hline \hline \multirow{3}{*}{ Hydrate } & Region & $\begin{array}{c}\text { Intervals } \\
(\mathrm{K})\end{array}$ & $\begin{array}{c}\text { Power } \\
(n)\end{array}$ \\
\hline \multirow{3}{*}{ xenon } & I & $2-6$ & $1.4 \pm 0.21$ \\
& II & $6-55$ & $0.3 \pm 0.1$ \\
& III & $55-97$ & $-1.9 \pm 0.2$ \\
methane & IV & $>97$ & $0.7 \pm 0.1$ \\
& I & $2-6$ & $1.2 \pm 0.2$ \\
& II & $6-54$ & $0.35 \pm 0.1$ \\
& III & $54-94$ & $-0.7 \pm 0.1$ \\
& IV & $>94$ & $0.2 \pm 0.1$ \\
\hline \hline
\end{tabular}

In the low temperature region (I) the temperature dependence of the thermal conductivity is not quadratic. The power $n$ is within $1.2-1.4$. Above $T_{1}=6 \mathrm{~K}$ (region II) the thermal conductivity depends weakly on temperature with $\kappa(T) \propto T^{0.3}$. The temperature regions, the interval III is of special interest. Here $\kappa(T)$ of Xe hydrate exhibits anomalous behavior - the thermal conductivity decreases by more than $50 \%$ as the temperature increases. An anomalous $\kappa(T)$ is also observed in $\mathrm{CH}_{4}$ hydrate in the similar interval, though in this case the anomaly is much less pronounced. The thermal conductivity decreases in the temperature region where the thermodynamic equilibrium phase with proton ordering may be present. $^{5}$

On completing the measurement, the Xe gas was removed from the Xe hydrate samples which then transform into ice. The effective thermal conductivity $\kappa_{\text {eff }}$ of grainy ice obtained through the hydrate-ice transformation was measured by the same procedure that was used to measure $\kappa_{\text {eff }}$ of gas hydrate. The results are shown in Fig. 1. The thermal conductivity of homogeneous ice was determined by Eq. (2). The results for two samples and also that for the sample obtained through $\mathrm{CH}_{4}$ hydrate dissociation ${ }^{6}$ are shown in Fig. 4, along with the thermal conductivity of bulk ice. ${ }^{23}$ The thermal conductivity of grainy ice is lower than that of bulk ice and is dependent on the history of the sample. This is accounted for by the large number of defects that appear in grainy ice due to gas hydrate dissociation. In particular, defects appear due to the dissociation-induced phase transition from the cubic structure to the hexagonal one. The process of formation of defects during gas-hydrate dissociation has been investigated thoroughly in Ref. 24.

The thermal conductivity of grainy ice has the temperature dependence characteristic of strongly deformed finegrained crystal dielectrics. At the lowest temperatures of the experiment the dependence was $\kappa(T) \propto T,{ }^{3}$ which is typical of phonon scattering at boundaries. At temperatures above $30 \mathrm{~K}$, the thermal conductivity decreases monotonically with growing $T$ (Fig. 4). The values of the thermal resistance of bulk ice and grainy ice are well described using the Rayleigh thermal resistance allowing for the phonon scattering at point defects. ${ }^{6}$ Figure 4 shows the curve $\kappa(T)$ calculated for grainy ice. The curve was calculated within the Debye model taking 


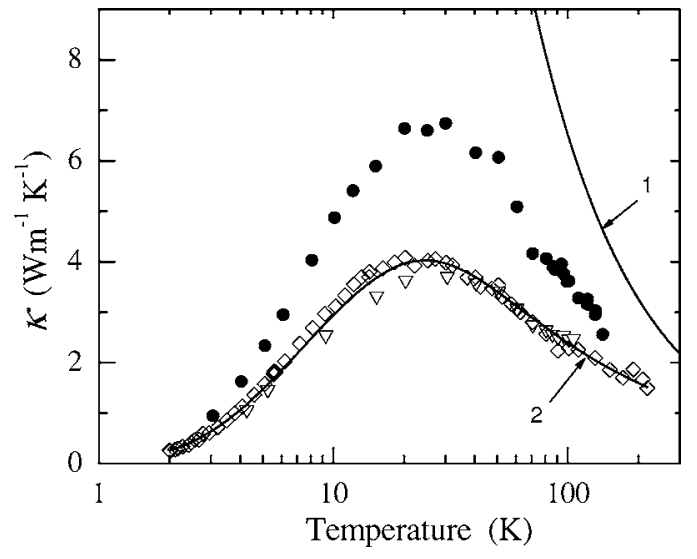

FIG. 4. Thermal conductivity of grainy ice resulting from dissociation of gas hydrates: $\mathrm{CH}_{4}$ hydrate $\diamond$ Ref. 6 ; Xe hydrate $\bigcirc ; \nabla$ Samples 1 and 2 accordingly. The solid line 1 represents thermal conductivity of perfect Ih ice crystal (Ref. 23). Solid line 2 is fitted $\kappa(T)$ for boundary and Rayleigh phonon scattering mechanisms (Ref. 6).

into account the additional mechanisms of phonon scattering (boundary and Rayleigh phonon scattering).

\section{DISCUSSION}

The temperature dependences of the thermal conductivity of clathrate hydrates obtained in this study are shown in Fig. 5 along with literature data for $\mathrm{CH}_{4},{ }^{6} \mathrm{THF},{ }^{7}$ 1,3-dioxolane. ${ }^{3}$ The comparison of the present and literature data reveals certain regularities in the behavior of the thermal conductivity of several gas clathrate hydrates. For example, at low temperatures (intervals I and II) the temperature dependence of clathrate hydrate is only weakly dependent on the type of clathrate structure. In these intervals the character of $\kappa(T)$ is practically independent of the nature of the guest molecules. In the whole range of temperatures $\kappa(T)$ the thermal conductivity of gas clathrate hydrates exhibits the glasslike behavior

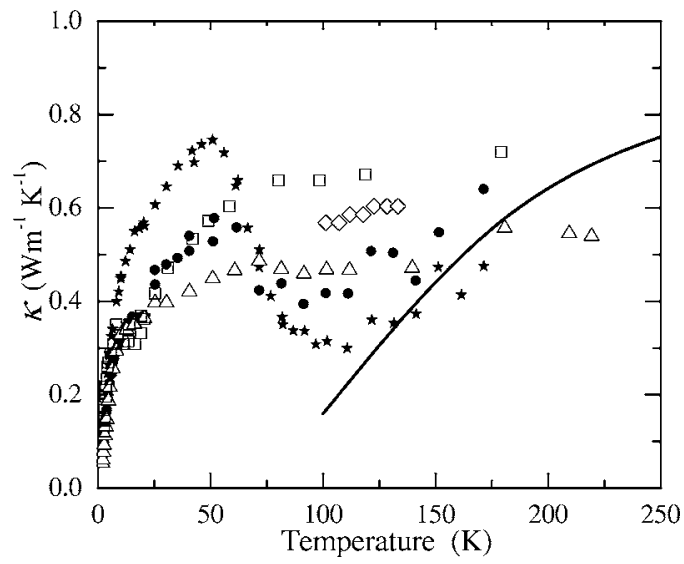

FIG. 5. Thermal conductivity for several clathrate hydrates: $\star$ Xe.6.2 $\mathrm{H}_{2} \mathrm{O}$, our data; $\bigcirc \mathrm{CH}_{4} \cdot 5.75 \mathrm{H}_{2} \mathrm{O}$ (Ref. 6); $\triangle \mathrm{THF} \cdot 16.9 \mathrm{H}_{2} \mathrm{O}$ (Ref. 7); $\square$ 1,3-dioxolane clathrate (Ref. 3); and $\diamond$ high density amorphous ice (Ref. 25). The solid line based on a MD simulation (Ref. 26) for Xe clathrate hydrate. typical for disordered solids. The exceptions are $\mathrm{Xe}$ and $\mathrm{CH}_{4}$ clathrate hydrates, whose thermal conductivity decreases with increasing temperature in interval III. At high temperatures (interval IV), $\kappa(T)$ is approximately proportional to $T$. Above $T \approx 75 \mathrm{~K}$, Xe clathrate hydrate has the lowest thermal conductivity of all investigated hydrates. At present there is no theoretical model permitting a quantitative description of the thermal conductivity of crystal clathrate hydrates in a wide region of temperatures.

Only in interval IV the experimental results for Xe clathrate hydrate agree rather well with the dependence calculated by molecular dynamics (MD) simulation. ${ }^{26}$ A possible mechanism of heat energy dissipation in clathrate hydrates is the strong coupling of local low-frequency translation vibrations of the guest and the acoustic phonons of the host lattice. $^{4,26-29}$

The information about the guest-host coupling was obtained in experiments investigating incoherent neutron scattering at the Type I structure of $\mathrm{CH}_{4}$ (Ref. 30) and Xe (Refs. 8,29 , and 30) clathrate hydrates. The mixing between the localized vibrations of the guest molecules (atoms) and the collective vibrations of the host lattice were observed in $\mathrm{Xe}$ and $\mathrm{CH}_{4}$ clathrate hydrates by the inelastic x-ray scattering technique. ${ }^{9}$ The mixing supports the idea of a resonant scattering mechanism.

In interval IV the propagation of thermal phonons becomes diffusive (Ioffe-Riegel mechanisms). ${ }^{31}$ The mean free path of phonons becomes smaller than the unit cell size. ${ }^{7}$ The heat spreads at the expense of energy transfer by direct collisions of atoms or molecules, which is responsible for the linear dependence of $\kappa(T)$ and explains the very close agreement between thermal conductivities of gas clathrate hydrates and high density amorphous ice. ${ }^{25}$

In most clathrate hydrates energy can be transferred by both translational and rotational motions of host and guest molecules. Guest molecules can contribute additionally to the heat transfer by scattering due to their rotational motion. This enables us to speculate as concerns the difference between $\mathrm{CH}_{4}$ and $\mathrm{Xe}$ hydrate. The picture changes at low temperatures (intervals I and II) when the thermal conductivity of the Xe clathrate hydrate exceeds that of clathrate hydrates with molecular guests. At low temperatures the heat is mainly transferred by long wavelength phonons. At the same time, librations can scatter phonons ${ }^{32}$ quite intensively and thus suppress the thermal conductivity of clathrate hydrates with molecular guests.

For all gas clathrate hydrates the glasslike behavior of $\kappa(T)$ in intervals I and II is well described by the phenomenological soft-potential model. ${ }^{33,34}$

The anomaly in the thermal conductivity in region III has been observed in several semiconductor clathrates, e.g., $\mathrm{Eu}_{8} \mathrm{Ga}_{16} \mathrm{Ge}_{30}$ and $\mathrm{Sr}_{8} \mathrm{Ga}_{16} \mathrm{Ge}_{30} \cdot{ }^{35}$ This feature in the thermal conductivity is indicative of the reduction in the resonant scattering contribution to thermal resistivity at low temperature. From a previous neutron scattering study of Xe clathrate hydrate $^{28}$ it is found that the resonant scattering is the strongest at the "rattling" guest mode anticrossing at $3.97 \mathrm{meV}$. This frequency corresponds to a temperature of $46 \mathrm{~K}$. Therefore, it is not surprising that the thermal conductivity in fact increases at low temperature when the popula- 
tion of the "localized" rattling vibrations decreases. For semiconductor clathrates, the same anomaly occurred at $15-20 \mathrm{~K}$. The lower temperature is probably due to the lower frequencies of the rattling motions of the guest atom as compare to those of $\mathrm{Xe}$ or $\mathrm{CH}_{4}$.

\section{CONCLUSION}

The thermal conductivity of $\mathrm{Xe} \cdot z \mathrm{H}_{2} \mathrm{O}$, where $z=5.9-6.2$, has been measured in a wide range of temperatures from $2 \mathrm{~K}$ to $170 \mathrm{~K}$ using the steady-state method. Four temperature intervals have been separated, in which the thermal conductivity has different temperature dependences. The behav- ior of $\kappa(T)$ in intervals I, II, and IV resembles the dependence typical for amorphous substances. $\kappa(T)$ is only weakly dependent on the type of clathrate structure or guest molecule. In the interval 56-97 K, where $\kappa(T)$ has an anomaly, the thermal conductivity dips over $50 \%$ when the temperature lowers.

\section{ACKNOWLEDGMENTS}

This work has been partly funded as a project by Forschungszentrum Jülich. We are grateful to Professor Werner Kuhs for preparation of the Xe clathrate hydrate material.
*Electronic address: krivchikov@ilt.kharkov.ua

${ }^{1}$ R. G. Ross, P. Andersson, and G. Backström, Nature (London) 290, 322 (1981).

${ }^{2}$ P. Andersson and R. G. Ross, J. Phys. C 16, 1423 (1983).

${ }^{3}$ N. Ahmad and W. A. Phillips, Solid State Commun. 63, 67 (1987).

${ }^{4}$ J. S. Tse and M. A. White, J. Phys. Chem. 92, 5006 (1988).

${ }^{5}$ O. Andersson and H. Suga, J. Phys. Chem. Solids 57, 125 (1996).

${ }^{6}$ A. I. Krivchikov, B. Ya. Gorodilov, O. A. Korolyuk, V. G. Manzhelii, H. Conrad, and W. Press, J. Low Temp. Phys. 139, 693 (2005).

${ }^{7}$ A. I. Krivchikov, V. G. Manzhelii, O. A. Korolyuk, B. Ya. Gorodilov, and O. O. Romantsova, Phys. Chem. Chem. Phys. 7, 728 (2005)

${ }^{8}$ B. Chazallon, H. Itoh, M. Koza, W. F. Kuhs, and H. Schober, Phys. Chem. Chem. Phys. 4, 4809 (2002).

${ }^{9}$ J. Baumert, C. Gutt, V. P. Shpakov, J. S. Tse, M. Krisch, M. Muller, H. Requardt, D. D. Klug, S. Janssen, and W. Press, Phys. Rev. B 68, 174301 (2003).

${ }^{10}$ J. S. Tse, K. Uehara, R. Rousseau, A. Ker, C. I. Ratcliffe, M. A. White, and G. MacKay, Phys. Rev. Lett. 85, 114 (2000).

${ }^{11}$ B. C. Sales, B. C. Chakoumakos, R. Jin, J. R. Thompson, and D. Mandrus, Phys. Rev. B 63, 245113 (2001).

${ }^{12}$ A. Bentien, M. Christensen, J. D. Bryan, A. Sanchez, S. Paschen, F. Steglich, G. D. Stucky, and B. B. Iversen, Phys. Rev. B 69, 045107 (2004).

${ }^{13}$ M. Zakrzewski and M. A. White, Phys. Rev. B 45, 2809 (1992).

${ }^{14}$ D. Michalski and M. A. White, J. Phys. Chem. 99, 3774 (1995).

${ }^{15}$ A. Missenard, Conductivite Thermique des Solides, Liquides, Gaz. et de Leurs Melanges (Editions Eyrolles, Paris, 1965).

${ }^{16}$ A. I. Krivchikov, B. Ya. Gorodilov, and O. A. Korolyuk, Prib. Tekh. Eksp. 48, 2 (2005).

${ }^{17}$ D. Davidson, in Gas Hydrates as Clathrate Ices, Natural Gas Hydrates-Properties, Occurrence and Recovery, edited by J.
Cox (Butterworth, Woburn, MA, 1983), pp. 1-16.

${ }^{18}$ G. N. Dulnev and Yu. P. Zarichnyak, Heat Conductivity of Mixtures and Composite Materials (Energiya, Moscow, 1974).

${ }^{19}$ A. R. Sanchez, Hans-Peter Klein, and M. Groll, Int. J. Hydrogen Energy 28, 515 (2003).

${ }^{20}$ Y. P. Handa and J. G. Cook, J. Phys. Chem. 91, 6327 (1987).

${ }^{21}$ J. G. Cook and D. G. Leaist, Geophys. Res. Lett. 10, 397 (1983).

${ }^{22}$ W. F. Waite, B. J. deMartin, S. H. Kirby, J. Pinkston, and C. D. Ruppel, Geophys. Res. Lett. 29, 2229 (2002).

${ }^{23}$ V. F. Petrenko and R. W. Whitworth, Physics of Ice (Oxford University Press, New York, 1999), p. 373.

${ }^{24}$ W. F. Kuhs, G. Genov, D. K. Staykova, and T. Hansen, Phys. Chem. Chem. Phys. 6, 4917 (2004).

${ }^{25}$ O. Andersson and H. Suga, Phys. Rev. B 65, 140201(R) (2002).

${ }^{26}$ R. Inoue, H. Tanaka, and K. Nakanishi, J. Chem. Phys. 104, 9569 (1996).

${ }^{27}$ J. S. Tse, V. P. Shpakov, V. V. Murashov, and V. R. Belosludov, J. Chem. Phys. 107, 9271 (1997).

${ }^{28}$ J. S. Tse, V. P. Shpakov, V. R. Belosludov, F. Trouw, Y. P. Handa, and W. Press, Europhys. Lett. 54, 354 (2001).

${ }^{29}$ J. S. Tse, C. I. Ratcliffe, B. M. Powell, V. F. Sears, and Y. P. Handa, J. Phys. Chem. A 101, 4491 (1997).

${ }^{30}$ C. Gutt, J. Baumert, W. Press, J. S. Tse, and S. Janssen, J. Chem. Phys. 116, 3795 (2002).

${ }^{31}$ D. G. Cahill and R. O. Pohl, Annu. Rev. Phys. Chem. 39, 93 (1988).

${ }^{32}$ Physics of Cryocrystals, edited by V. G. Manzhelii and Yu. A. Freiman (AIP-Press, Woodbury, New York, 1996).

${ }^{33}$ U. Buchenau, Yu. M. Galperin, V. L. Gurevich, D. A. Parshin, M. A. Ramos, and H. R. Schober, Phys. Rev. B 46, 2798 (1992).

${ }^{34}$ M. A. Ramos and U. Buchenau, Phys. Rev. B 55, 5749 (1997).

${ }^{35}$ J. L. Cohn, G. S. Nolas, V. Fessatidis, T. H. Metcalf, and G. A. Slack, Phys. Rev. Lett. 82, 779 (1999). 